\title{
Plastics give and plastics take
}

\author{
Plastics have profoundly changed what is possible in modern society. But between their reliance \\ on fossil fuels and their massive accumulation as waste, plastics are also at the heart of a dual \\ environmental crisis.
}

Plastic waste has been a top public concern for decades. In 1984, the New York Times reported on a "new and insidious form of pollution" in our oceans. Generations of kids have been taught to worry about sea turtles caught in plastic six-pack rings, a visceral reminder of what plastic waste can do to ecosystems.

But pollution is not plastics' only threat to the environment. The vast majority of plastics are made from fossil fuels, and every stage of the plastic life cycle from extracting resources and refining chemicals, to recycling and incinerating - pumps greenhouse gases into the atmosphere. As demand for plastics continues to rise, it is estimated that by 2050 plastics and other petrochemicals will account for nearly half of growth in oil demand and drain up to 15\% of our global carbon budget. Even as the energy and transportation sectors move away from fossil fuels after hard-fought battles, plastics are poised to undermine the progress.

As materials science editors, we are awed by the power of plastics to shape the modern world, but also by the scale of the crisis they have created. In this month's Focus Issue, we feature articles that analyse the problem of plastics from many perspectives.

To remove reliance on fossil fuels, polymers can be made from renewable bio-based resources such as plants. How sustainable and commercially viable this strategy is remains an open question: Giovanni Traverso and co-authors examine whether biomass-derived plastics, for all their promise, are actually compatible with all stages of a circular plastics economy. Eugene Chen and colleagues contend that bio-based polymers, to be competitive with fossil-based standards, must offer performance advantages that arise from the distinct chemical structure of the bio-feedstock. Swapping petroleum-derived compounds for chemically identical but biogenic replacements is not likely to crack the established market.

Fossil-based polymers can also be produced more sustainably through green syntheses that achieve high atom economy and energy efficacy under mild reaction conditions. As Min Sang Kwon and colleagues propose, visible light has potential as a renewable and abundant energy source to polymerize plastics.

To reduce plastic pollution in the short term, though, we urgently need to improve the recyclability and waste management of existing products. Recycling is hardly the solution it is heralded to be, at least yet; only $\sim 9 \%$ of all global plastic waste has been recycled. Every step of recycling costs energy, cuts plastic quality and loses material, as Vânia Zuin and Klaus Kümmerer write, and circulation processes such as downcycling simply postpone the problem to the future. Similarly, Denise Mitrano and Martin Wagner urge us to rethink our relationship with plastics, highlighting that we need to make large-scale changes to the way we make, use and dispose of plastics to mitigate plastic pollution. They advise that materials and products should be designed as simply as possible, making them easier to separate and recycle. However, designing a new polymer with end-of-life management in mind is a system-wide effort that requires collaboration between not only polymer chemists, but also environmental engineers and waste treatment professionals, as Kara Lavender Law and Ramani Narayan discuss. Any new design must include a plan for the polymer's recovery that is compatible with a region's existing recycling infrastructure.

When plastic waste is mismanaged, it enters our environment. In 2001, the European Environmental Agency declared early warning signs - including persistency, widespread distribution and potentially irreversible harm - that should ring alarm bells in regulators when evaluating any new technology for potential risk. They were not referring to plastics specifically, but Steffen Hansen and colleagues point out that the warning signs are indeed relevant to plastics. For example, the same chemical stability that makes plastics so useful also makes them annoyingly persistent pollutants. And as plastics break down into smaller particles, they have the potential to harm a wider range of species through an increasing variety of pathways. But microplastics are extraordinarily complex, and their risks are still scantily understood. To help understand these risks in a regulatory capacity, Albert Koelmans and co-authors outline a risk assessment framework for microplastics that takes into account their multidimensionality.

The problem of plastics is profoundly complex. One theme is clear: all relevant research disciplines and sectors of society must collaborate to find solutions that address the full plastics life cycle. As we assemble this Issue, we are energized by the ingenuity of this month's authors and their commitment to confronting the crisis. 